\title{
An Experiment Study on Performance Evaluation of Black-Body System by using Infra-Red Camera
}

\author{
by Seoktae. Yoon* and Yongjin. Cho**
}

\author{
* Pusan National Univ. of Engineering, 2, Busandae 63 Str., Busan, Republic of Korea, yst0531@pusan.ac.kr \\ ** Dong-Eui Univ. of Engineering, 176, Eomgwang Str., Busan, Republic of Korea, cyjdeu@deu.ac.kr
}

\begin{abstract}
The blackbody system is an optical device developed based on the basic concept of blackbody. A blackbody is an ideal object that absorbs all the energy entering the surface from surrounding objects and emits maximum energy at a given temperature and wavelength. Infrared cameras measure the temperature of object in a non-contact method and perform calibration by using a blackbody system. Therefore, it is necessary to evaluate and preserve the performance of the blackbody system itself before using to calibrate other optical equipment. In this study, we used an infrared camera that was calibrated by a high reliability organization for calibration, and conducted an experimental study to check the preservation of system performance by measuring before and after calibration of the blackbody system. The blackbody system uses extended type, and uses the noise equivalent temperature difference and three-dimensional noise of the infrared camera detector as an index for evaluating the performance of the blackbody system.
\end{abstract}

\section{Background}

Various instrument using optical characteristics are being developed due to the enhancement of modern physics. Representative optical instrument is laser system, thermography and black-body system among which black-body system is based on the concept of black-body law. A blackbody is an ideal object that absorbs all the energy entering the surface from surrounding objects and emits maximum energy at a given temperature and wavelength. Many modern optical instrument use black-body system annually to perform calibration. Therefore, important things is preserve the performance of the black-body system. In this paper, we used an infrared camera that was calibrated by a high reliability organization for calibration, and conducted an experimental study to check the preservation of system performance by measuring before and after calibration of the blackbody system. The blackbody system uses extended type, and uses the noise equivalent temperature difference and three-dimensional noise of the infrared camera detector as an index for evaluating the performance of the blackbody system.

\section{Experiment}

We used extended type black-body system that controls the surface temperature as the oil circulate. Therefore, if we increase the setting temperature of black-body system, heat energy is concentrated in the center of the surface.

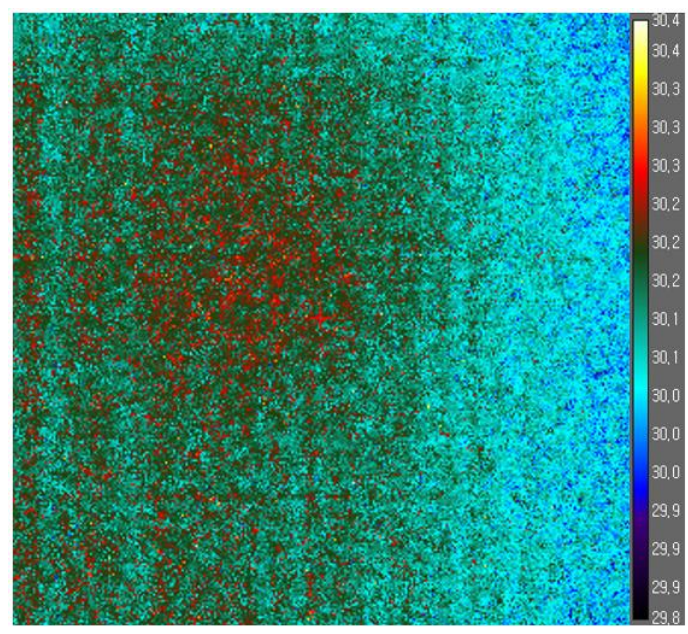

Set temperature of black-body system : $30^{\circ} \mathrm{C}$

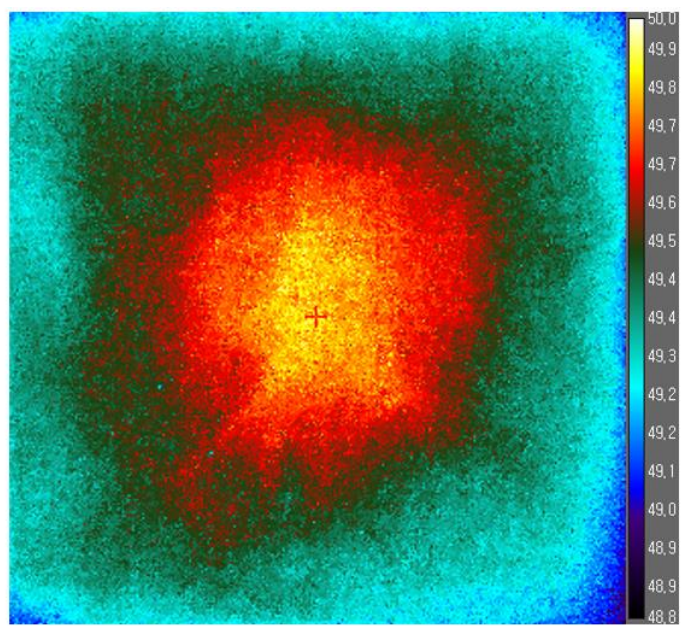

Set temperature of black-body system : $50^{\circ} \mathrm{C}$

Figure 1 Thermal energy distribution 
Figure 1 is an image of heat energy distribution on the blackbody surface at $30^{\circ} \mathrm{C}$ and $50^{\circ} \mathrm{C}$. This shows that if we setting more high temperature, heat energy is concentrated in the center of blackbody surface. Therefore, the experimental conditions were set after check the heat energy uniformity of the blackbody system was changed by $2^{\circ} \mathrm{C}$. The result is shown in Figure 2.

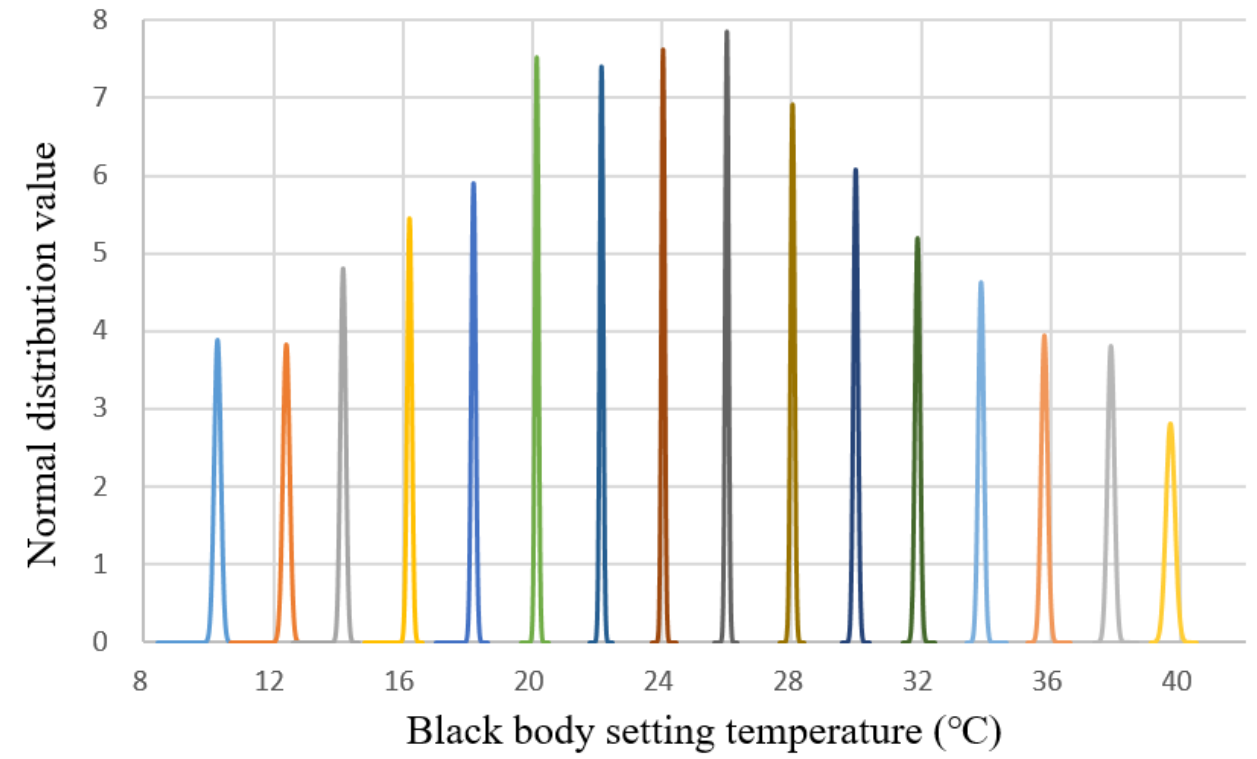

Figure 2 Analysis surface temperature uniformity of blackbody system

In Figure 2, the temperature of the blackbody system is used from 20 to 30 degrees so that 25 is center. In addition, the shield was installed during set up process to prevent heat energy disturbance around.

\section{Result}

To verify the performance of the blackbody system, NETD results and 3D noise were calculated and the results are shown in Figure 3,4. The results show that the spatial noise of the blackbody system changes. The reason for this is that the oil residue on the surface of the blackbody system is more noise.

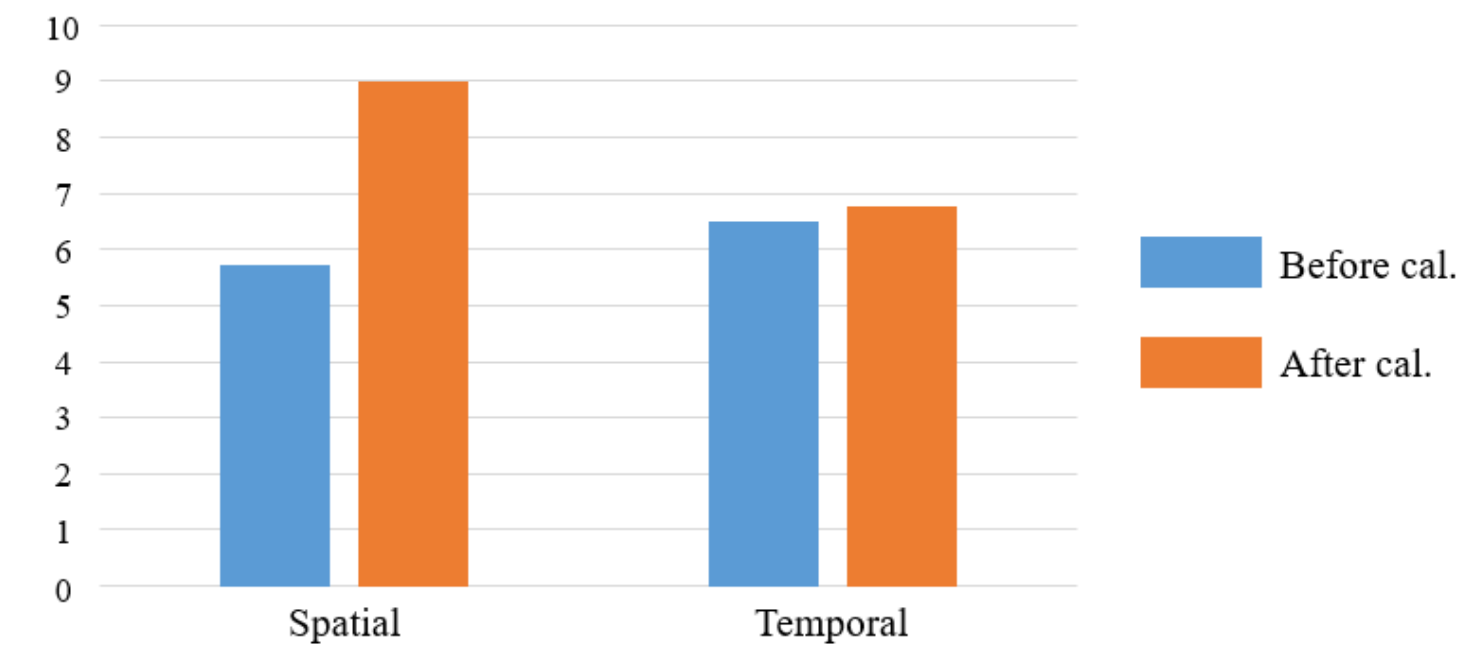

Figure 3 NETD result of LWIR camera 


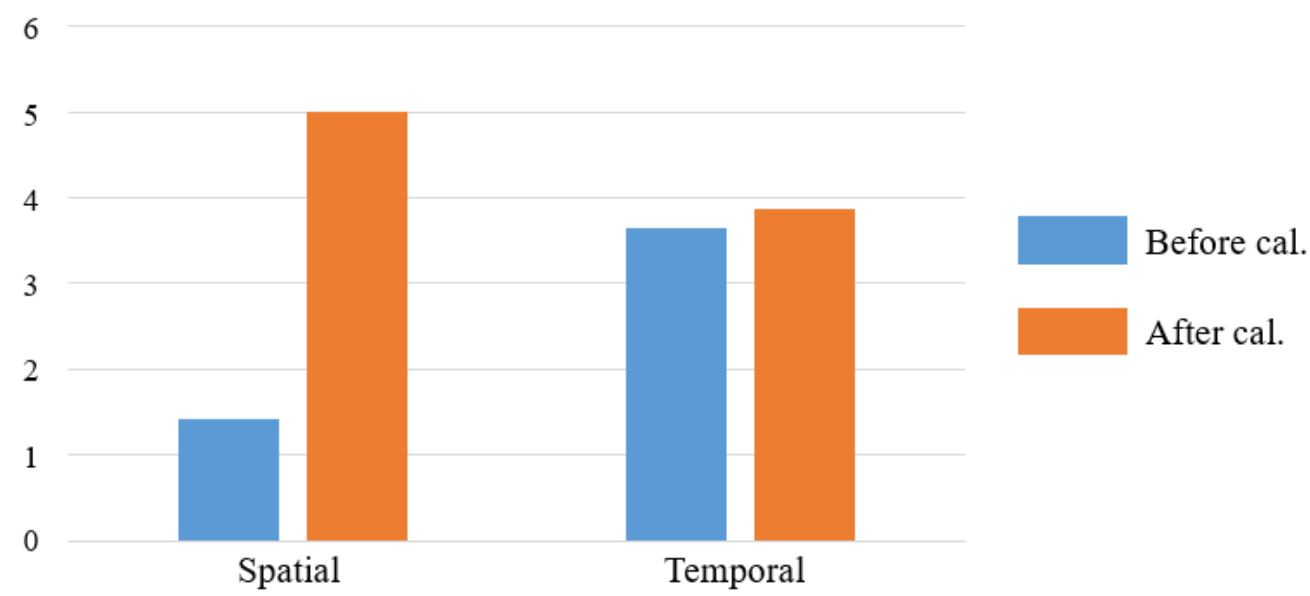

Figure 4 NETD result of MWIR camera

The 3D noise results measured using long-wave infrared (LWIR) and Middle-wave infrared (MWIR) cameras are shown in Figs. 5 and 6, respectively. Detectors of LWIR camera visualize thermal energy in response to wave energy. On the other hand, Detectors of MWIR cameras visualize thermal energy in response to quantum energy. As a result, the MWIR camera reacts more sensitively, changing the result of 3D noise.

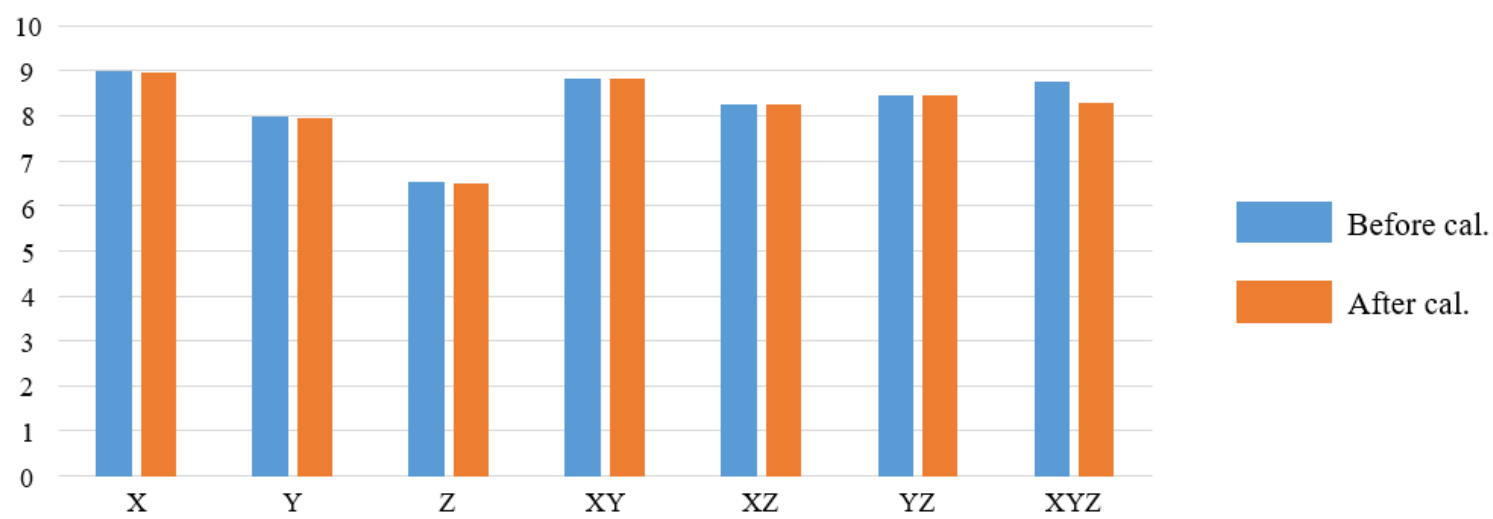

3D noise result of LWIR camera

\section{Figure 5 3D noise result of LWIR camera}

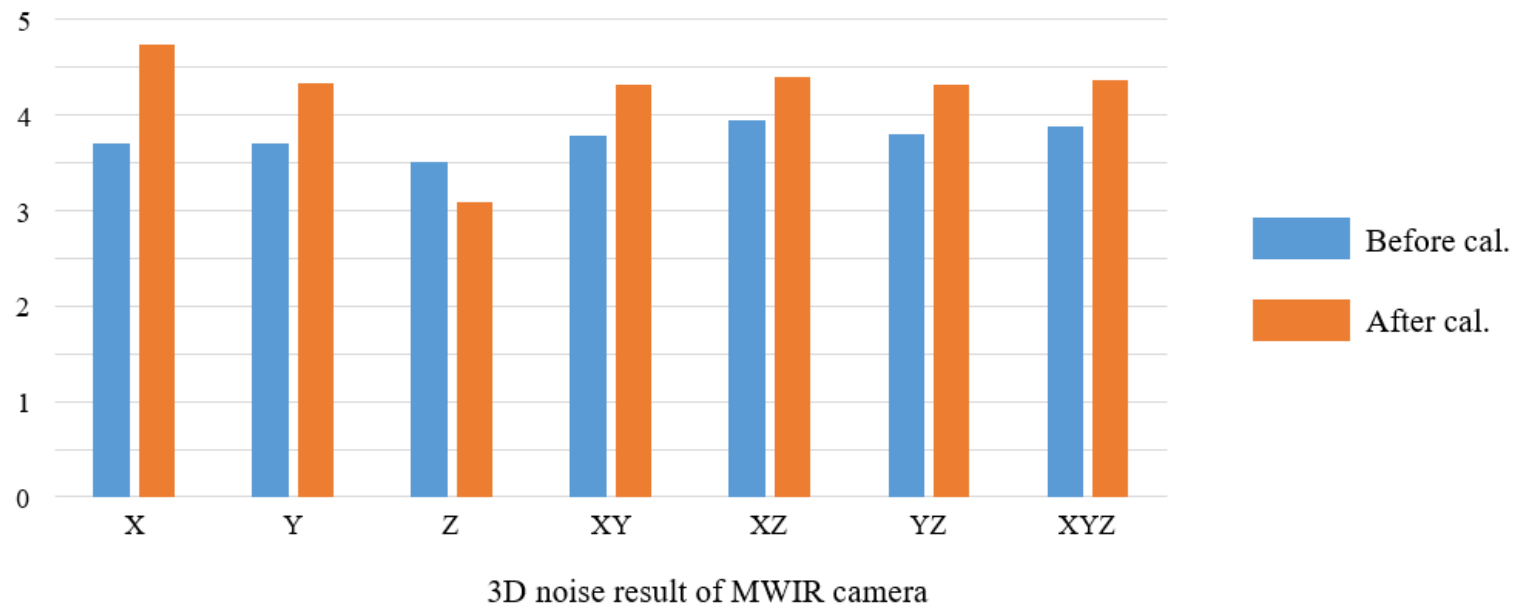

Figure 6 3D noise result of MWIR camera 


\section{Conclusion}

In this study, the blackbody system was evaluated using the verified thermography system. For this purpose, we check the operating characteristics of the blackbody system to setup experiment condition. The index for evaluating the blackbody system is the noise equivalent temperature difference and three-dimensional noise of the thermograph system. In the case of the NETD results, the spatial noise increased in both the LWIR and MWIR cameras. For 3D noise results, only the MWIR camera with better detector performance changed.

\section{REFERENCES}

[1] D.I. Kim, G.S. Kim, G.H. Kim, K.S. Chang "Responsivity and Noise Evaluation of Infrared Thermal Imaging Camera". Journal of the Korean Society for Nondestructive Testing, vol. 33, pp.342-348, 2013.

[2] S.U. Kim, M.Y. Choi, J.H. Park, K.Y. Shin, E.C. Lee, "Development of Calibration Target for Infrared Thermal Imaging Camera". Journal of the Korean Society for Nondestructive Testing, vol. 34, pp.248-253, 2014. 